\title{
Dent in Material
}

National Cancer Institute

\section{Source}

National Cancer Institute. Dent in Material. NCI Thesaurus. Code C63245.

Problem associated with a undesired change in shape, characterized by the presence of a slight hollow (dent) in the device surface. 